\title{
New Synthesis: The Evolutionary Ecology of Floral Volatiles
}

\author{
Amy Parachnowitsch
}

Published online: 30 August 2014

(C) Springer Science+Business Media New York 2014

\begin{abstract}
Natural selection is a key process of population to species divergence and diversification. Since Lande and Arnold's 1983 seminal paper "The measurement of selection on correlated characters", hundreds of evolutionary biologists have measured phenotypic selection in wild populations (Kingsolver et al. 2012). These microevolutionary studies aim to understand the process of adaptive evolution, by regressing variation in putatively adaptive traits against measurements of fitness. The LandeArnold approach can compare selection strength, direction, and shape for different traits, making it a powerful tool for studying contemporary evolution. Although well over 50 studies have measured selection on floral characters, until recently none have incorporated chemical features of floral phenotypes, such as scent. Differing traditions may in part explain the scarcity of selection studies on floral scent; while evolutionary ecologists have focused on visual and mechanical aspects of floral display, floral scents have been more commonly studied in functional and ecological contexts. However, integration of floral characters into a unified perspective is critical to understand both function and evolution of floral diversity.
\end{abstract}

Two pieces of information are needed to measure phenotypic selection: 1) trait(s) that vary among individuals (ideally heritable variation rather than environmental or measurement factors) and 2) a fitness outcome that can be linked to those traits. Neither of these criteria should limit studying selection on floral scents; improved techniques allow for large-scale field sampling of volatiles, and fitness can be measured as survival, seed production, or genetic estimates of paternity. Once data are gathered, statistical models that calculate multivariate selection gradients ( $\beta$ ) control for correlations among traits to determine direct targets of selection. However, these models are only as good as the traits included, therefore, an important caveat is that selection targets may instead be some unmeasured trait. Because floral volatiles have been largely ignored in selection studies, there is a real possibility that our understanding of floral evolutionary ecology lacks the true targets of selection. For example, frequently observed selection on corolla size could be due to its role as a visual signal or as a mechanical landing platform for pollinators as often hypothesized, or as its role as a scent-emitting surface. Alternatively, unlike many other floral characters, volatiles may not relate to fitness if they are too dynamic in nature for individuals to exhibit consistent phenotypes. However, without data it is difficult to construct general conclusions.

Recent studies have demonstrated the possibility to measure phenotypic selection on floral scents (e.g., Schiestl et al. 2011). However, a challenge is to rigorously define the trait or phenotype. Although floral scents are generally constitutively expressed, in contrast to herbivoreinduced volatiles, floral scents can vary through space and time, due to physiological factors, abiotic conditions, and ecological interactions.

\footnotetext{
A. Parachnowitsch $(\bowtie)$

Department of Plant Ecology, Evolutionary Biology Centre,

Uppsala Universitet, Uppsala, Sweden

e-mail: amy.parachnowitsch@ebc.uu.se
}

Moreover, scent bouquets often are complex blends of many compounds where abundant compounds are not necessarily the most important in ecological interactions. Thus, exploring scent data through statistical techniques and ecologically relevant handling of scent blends (e.g., antennally active vs. non-active scents, (Schiestl et al. 2011)) will enhance our ability to detect selection on this complex trait.

However, those measuring selection on scents should avoid following the trajectory of early microevolutionary ecologists by simply relating traits to fitness. Although that approach has yielded much, its limitations are also apparent (Kingsolver et al. 2012). In particular, knowing selection on a trait can inform its predicted evolution but does not address why selection occurs. Unfortunately, equating preferences of interacting organisms to agents of natural selection can lead to false conclusions. For example, pollinators may prefer larger or more strongly scented flowers, and it is tempting to assume that selection to increase these traits is due to their preference. However, selection exerted via biotic interactions will depend on their strength. For pollinator-mediated selection this means pollinator behavior can drive selection only when non-preferred floral phenotypes receive insufficient visitation for reproduction (e.g., Galen et al. 2011). Further, pollinators are one among many potential selective agents of flowers, including antagonists and abiotic conditions. The causes of natural selection can be determined only by explicitly examining agents, preferably by experimental manipulation.

Integrating floral chemical ecology and evolutionary ecology can answer such questions as: Is selection on floral volatiles common? How does selection on floral volatiles compare to visual/mechanical traits? Does selection act on signals independently or as integrated phenotypes of visual and olfactory signals? Further, selection studies can act as a filter for determining key volatiles from a blend, where targets of selection from a bouquet can be examined for functional roles in ecological interactions. Exploring both natural selection and ecological functions aids interpreting the vast diversity of floral characters across angiosperms but investigating different aspects of flowers in isolation will hamper our understanding of both the functional roles and evolution of these complex traits.

\section{References}

Galen C, Kaczorowski R, Todd SL, Geib J, Raguso RA (2011) Dosagedependent impacts of a floral volatile compound on pollinators, larcenists, and the potential for floral evolution in the alpine skypilot Polemonium viscosum. Am Nat 177:258-272

Kingsolver J, Diamond S, Siepielski A, Carlson S (2012) Synthetic analyses of phenotypic selection in natural populations: lessons, limitations and future directions. Evolut Ecol 26:1101-1118

Schiestl FP, Huber FK, Gómez JM (2011) Phenotypic selection on floral scent: trade-off between attraction and deterrence? Evolut Ecol 25: 237-248 